\title{
High precision dual-modulation differential terahertz ATR sensor for liquid measurements
}

\author{
Xiujun Zheng, ${ }^{1}$ Thomas Gevart, ${ }^{1}$ And Guilhem Gallot $1,{ }^{1}$ \\ ${ }^{1}$ Laboratory for Optics and Biosciences, Ecole polytechnique, CNRS, INSERM, IP Paris, Palaiseau, France \\ *Guilhem.Gallot@polytechnique.edu
}

\begin{abstract}
We describe a highly sensitive and stable QCL-based attenuated total reflection terahertz sensor for the detection of very low concentration solutions, using a dual modulation differential approach and ATR geometry. This sensor offers a very high dynamic range and a long-term stability of $40 \mathrm{~dB}$, which extends the potential of terahertz radiation for the analysis of liquid and biological samples. The performance is illustrated by measurements on standard solutions of ions, sugars and proteins, for concentrations down to $1 \mu \mathrm{M}$.
\end{abstract}

(C) 2021 Optical Society of America

Terahertz sensing has become a proven tool for the non-destructive analysis of materials in the frequency range of $0.1 \mathrm{THz}$ to $5 \mathrm{THz}$. Gaseous and solid samples have been widely studied as they exhibit resonant spectral lines and relatively low absorption. Liquids, on the contrary, have much broader featureless spectral structures and are highly absorbent, especially polar liquids, so that optical constants are difficult to measure [1]. However, polar liquids such as water play a central role in chemical physics and biology [2]. The hydration shell, which is the water interface around any solute, is for instance of major importance in biochemistry for protein activity [3]. The detection of small variations in the terahertz properties of solutes would also benefit the study of living cells, which have a terahertz dielectric constant difference of a few percent between the inside of the cell and its surrounding medium. [2,4-6]. However, since water absorption is high, these small variations are often hidden by the instability of the sensor.

To measure the terahertz dielectric properties of liquids, many techniques have been used: Time-Domain Spectroscopy [7-9], P-germanium laser [10], spoof plasmons [11], ATR [12-15], synchrotron radiation [16], etc. To detect very low concentration levels or very small sample volumes, many types of sensors have been demonstrated: meta-atoms [17], microfluidics [18], gratings [19], chemical sensing plate [20], Fabry-Pérot [21], ring resonators [22], etc. Particular attention must also be paid to long-term stability, especially for studies on biological systems [4-6]. Here, we present an attenuated total reflection (ATR) terahertz sensor with very high detection sensitivity and long-term stability, based on a dual-modulation approach, for liquid and cellular samples.

When light is reflected at the interface of two optical media with refractive indices $n_{1}>n_{2}$, with incidence angle $\theta$, total reflection occurs for $n_{1} \sin \theta>n_{2}$ and an evanescent field exists in the optically less dense medium $n_{2}$. If an absorbent layer is put on top of the prism, it couples with the evanescent field and decreases the amount of reflected energy, leading to ATR [12]. The measure of the reflected beam is then directly correlated with the complex dielectric constant of the absorbing medium covering the prism. As the penetration depth is a few tens of micrometers in the terahertz domain, the height of the liquid at the top of the prism has no influence. We have designed an original dual-modulation ATR setup, based on a synchronous optical modulation of the terahertz beam, one half for sample measurement and the other for reference, using a symmetrical sample/reference optical path through the ATR prism, and a single detector.

The setup is based on a terahertz Quantum Cascade Laser (QCL) source (TeraCascade 1000, Lytid) emitting $3 \mathrm{~mW}$ in $\mathrm{CW}$ at $2.5 \mathrm{THz}$. The vertically polarized divergent output beam is collimated by a $f=50 \mathrm{~mm}, D=50 \mathrm{~mm}$, high-resistivity silicon (HR-Si) lens. The beam is then 

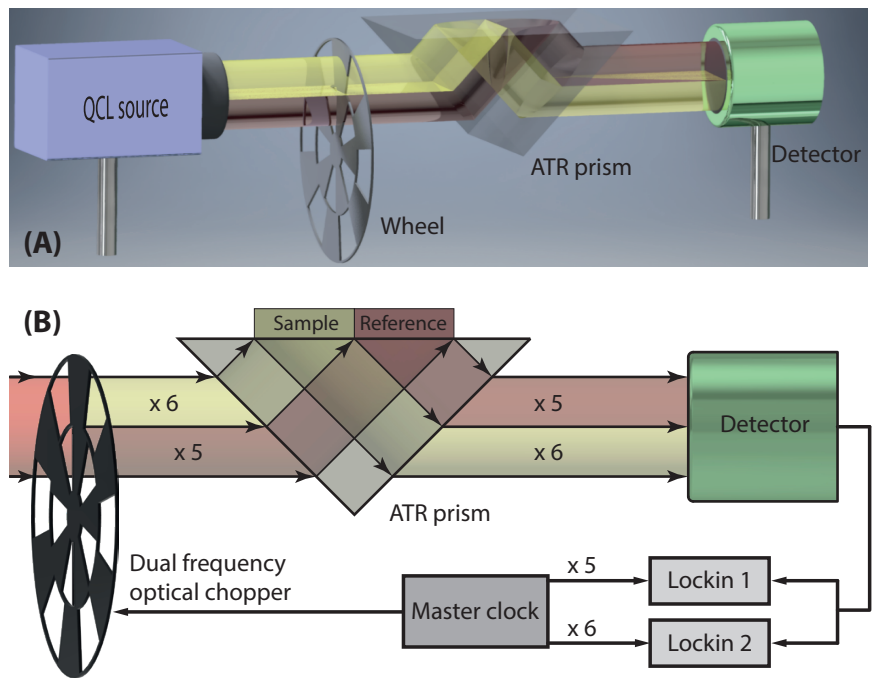

Fig. 1. Dual-modulation differential ATR sensor setup. (A) 3D view. (B) Detailed view. The terahertz beam from the QCL source is chopped at two different frequencies $\times 5$ and $\times 6$ by the two rows of chopper slots. Half of the beam is modulated at $\times 6$ and is reflected by the sample while the other is modulated at $\times 5$ and used as a reference. The two halves are recombined and sent to the same detector. Two lock-in amplifiers are used to detect the sample and reference signals. The chopper controller provides the modulation signals for the optical chopper and lock-in amplifiers.

reduced to a 4-mm full width at half maximum (FWHM) collimated beam using an afocal pair consisting of an off-axis parabolic mirror $(f=150 \mathrm{~mm})$ and a HR-Si lens $(f=25 \mathrm{~mm})$, with a reduction ratio of 6 . All HR-Si lenses are AR-treated at $2.5 \mathrm{THz}$ to reduce reflection loss. The beam is then directed to the ATR sensor described in Fig. 1. First, the beam passes through a dual frequency optical chopper and is simultaneously chopped at two different frequencies. The upper half of the beam is chopped by 6 outer slots, while the lower half is chopped by 5 inner slots. Thus, the upper and lower halves of the beam are modulated, relatively to a master frequency set at about $65 \mathrm{~Hz}$, at frequencies of $\times 6(390 \mathrm{~Hz})$ and $\times 5(325 \mathrm{~Hz})$, respectively. Both halves then undergo an ATR on the HR-Si prism $\left(50 \times 50 \mathrm{~mm}\right.$ input face, angle of $\left.42^{\circ}\right)$ at two separate positions, where the sample and reference liquids can be placed. After exiting the prism, both parts of the parallel beam are focused on a pyroelectric detector with a surface area of $4 \mathrm{~mm}^{2}$ (TeraPyro, Lytid, $390 \mathrm{~V} / \mathrm{W}$ sensitivity) by a HR-Si lens $(f=25 \mathrm{~mm})$. The pyroelectric signal, resulting from the superposition of the two modulations, is demodulated by two independent lock-in amplifiers driven by each of the two frequencies provided by the chopper controller. One obtains the signals $S_{5}(\times 5)$ and $S_{6}(\times 6)$ for both modulations. The whole setup is placed on a water-cooled breadboard, and in a tight box with controlled humidity (below $2 \% \mathrm{RH}$ ) and temperature $\left(21 \pm 0.2^{\circ} \mathrm{C}\right)$. Since the dielectric constant of liquids is sensitive to temperature (for instance for water, a variation of $1^{\circ} \mathrm{C}$ corresponds to a variation of ATR signal of $0.5 \%[23,24]$ ), the ATR prism itself is precisely thermalized to obtain a liquid temperature stability of $\pm 0.01^{\circ}$ using two Peltier thermoelectric coolers (TEC) and a Thorlabs TED200C temperature controller. The ATR sensor is thus characterized by excellent signal-to-noise ratios at short-term $(32 \mathrm{~dB}$, $300 \mathrm{~ms})$, medium-term $(40 \mathrm{~dB}, 10 \mathrm{~s})$ and long-term ( $30 \mathrm{~dB}$ over hours). The latter is less good than at $10 \mathrm{~s}$ because it has to compensate for the fluctuations of the whole system and not only of the source and detector. However, it is essential for long-term analyses, especially for dynamic monitoring of biological samples. 

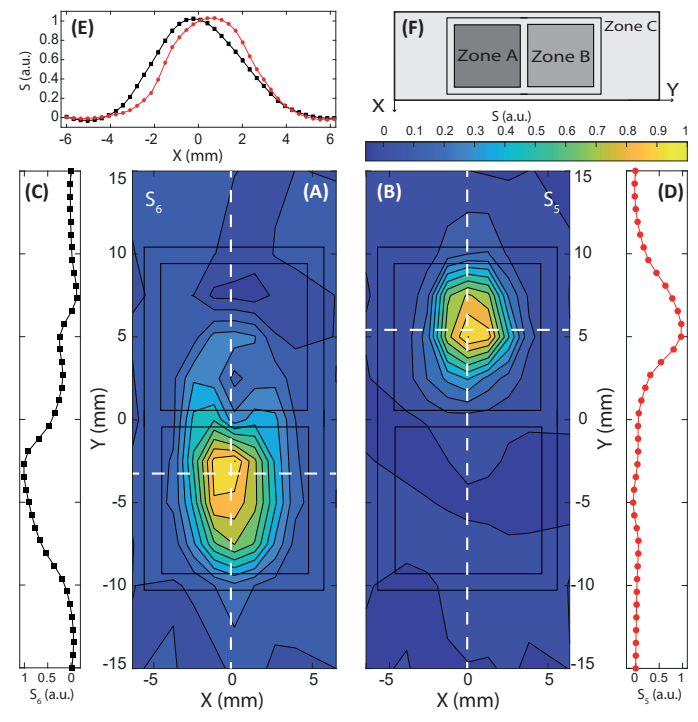

Fig. 2. Profiles of the terahertz signals of the ATR sensor on the HR-Si prism at the two modulation frequencies $\times 6$ and $\times 5$. The central figures show the $2 \mathrm{D}$ profiles of $S_{6}$ (A) and $S_{5}$ (B). The side figures (C) and (D) are cross sections along the Y-direction (direction of propagation), and figure (E) shows the cross sections along the $\mathrm{X}$-direction (transverse to propagation) for $S_{6}$ (black) and $S_{5}$ (red). Figure (F) is the geometry of the prism and the 3 zones including the liquid chambers for measurements and calibration. The dashed lines in figures (A) and (B) show the cross sections, and the rectangles represent the liquid chambers.

The spatial distribution of the terahertz ATR signal on the sensor prism is obtained by using a tiny $2 \mu \mathrm{L}$ drop of water directly on the prism. The addition of the drop locally decreases the S5 and S6 signals and allows the profiles of the two beams to be measured at the $\mathrm{x} 5$ and $\mathrm{x} 6$ modulations, respectively. The water drops are placed successively on the prism on a 23 by 31 grid evenly spaced by $2 \mathrm{~mm}$. For each position, the average of 5 consecutive measurements is taken and compared to the signal without water. The diameter of each drop is less than $2 \mathrm{~mm}$, which sets the spatial resolution. The result is given by Fig. 2, showing a very good localization of the two modulations. The profiles of the $\mathrm{X}$ and $\mathrm{Y}$ directions are of Gaussian form. This is expected since the Fresnel numbers in this configuration are of the order of unity, due to the very long wavelength $(\lambda=120 \mu \mathrm{m})$ [24]. This means that diffraction plays a major role in shaping the beams from the chopper to the prism and produces Gaussian-like profiles with partial overlap. Each distribution has a FWHM of about $4.4 \mathrm{~mm}$ along the X-direction. Along the Y-direction, FWHM is $5.4 \mathrm{~mm}$ for $S_{5}$ and $7.6 \mathrm{~mm}$ for $S_{6}$. Most importantly, we observe a partial overlap between the two distributions, which is explained by the significant diffraction created by the optics and especially the optical chopper, which broadens the beams. This has an important consequence on the calibration of the sensor.

Measurements of solutions are performed with a double liquid chamber plate put on top of the ATR prism. The double $10 \times 10 \mathrm{~mm}$ plastic chamber is glued to a $3 \mathrm{~mm}$-thick HR-Si plate, which can be positioned in close contact to the prism. Each chamber A and B can hold liquid samples or remain empty (see Fig. 2, upper right). The volume of liquids is about $1 \mathrm{~mL}$ to ensure covering of the whole surface and to minimize the influence of liquid evaporation. Zone $\mathrm{C}$ surrounding the chambers always remains empty. Since the flatness of the prism and plate surfaces is not perfect, tiny air gaps between the prism and plate can create a large reflection loss at the interfaces. To 
avoid this, we used $\alpha$-pinene (Sigma-Aldrich, P45702) as an index-matching liquid between the prism and plate. To compensate for the addition of the chamber plate, the position of the prism is moved down by $3 \mathrm{~mm}$ by a translation plate. The use of the extra plate and index-matching liquid results in a slight signal drop of a few percent compared to the prism alone. The main purpose of the sensor is to detect small variations of ATR reflectivity of solutions compared to pure water, in other words we want to measure the coefficient $\epsilon_{S}=\left(R_{S}-R_{W}\right) / R_{W}$, where $R_{S}$ and $R_{W}$ are the ATR reflectivity for solutions and pure water, respectively.

Due to the partial overlap between the two signal distributions, special care must be taken for calibration. The signals $S_{5}$ and $S_{6}$ come from the sum of the reflections of the 3 zones (A, B, C) on the prism (see Fig.2, upper right), and are modeled as

$$
S_{5 / 6}^{\mathrm{AB}}=\left[m_{5 / 6}^{\mathrm{A}} R_{\mathrm{A}}+m_{5 / 6}^{\mathrm{B}} R_{\mathrm{B}}+m_{5 / 6}^{\mathrm{C}}\right] \cdot T_{5 / 6} \cdot I_{0}
$$

where $I_{0}$ is the laser intensity at the sensor input, $T_{5 / 6}$ are the unknown transmission coefficients of the whole optical system without the prism, for the modulations $\times 5$ and $\times 6$, and $m_{5 / 6}^{Z}=$ $\int_{\mathcal{A}^{\mathrm{z}}} P_{5 / 6}(r) \mathrm{d} r$ are the overlap integrals between the profiles $P_{5 / 6}(r)$ measured on the prism for both modulations and the zone areas $\mathcal{A}^{\mathrm{Z}}$. To cancel the fluctuations of $I_{0}$, we routinely calculate, from the two measured lock-in amplifiers signals $S_{5}$ and $S_{6}$, the ratio

$$
r^{\mathrm{AB}}=S_{6}^{\mathrm{AB}} / S_{5}^{\mathrm{AB}}
$$

for the couple of solutions present in chambers A and B. However, the values of $T_{5 / 6}$ and $m_{5 / 6}^{\mathrm{Z}}$ remain unknown and calibration is required.

Since the addition of solutes has relatively little effect on the ATR coefficient of the solution compared to pure water [25], it is assumed that $\epsilon_{S} \ll 1$. After a simple calculation, we find that the desired quantity $\epsilon_{S}$ can be obtained from the experimental measurements $r^{\mathrm{SW}}$ and $r^{\mathrm{WW}}$ defined in Eq. 2 with $\mathrm{S}$ and $\mathrm{W}$ referring respectively to the studied Solution and pure Water in chambers A and B, such that

$$
\epsilon_{S}=\frac{1}{\beta}\left(r^{\mathrm{SW}}-r^{\mathrm{WW}}\right) / r^{\mathrm{WW}},
$$

where the calibration parameter $\beta$ is related to the ATR profiles as

$$
\beta=R_{\mathrm{W}}\left[\frac{m_{6}^{\mathrm{A}}}{1+\left[m_{6}^{\mathrm{A}}+m_{6}^{\mathrm{B}}\right]\left(R_{\mathrm{W}}-1\right)}-\frac{m_{5}^{\mathrm{A}}}{1+\left[m_{5}^{\mathrm{A}}+m_{5}^{\mathrm{B}}\right]\left(R_{\mathrm{W}}-1\right)}\right]
$$

In the ideal case where each modulated beam does not extend beyond its allocated zone, the integrals $m_{5 / 6}^{\mathrm{Z}}$ are null except $m_{6}^{\mathrm{A}}=m_{5}^{\mathrm{B}}=1$, so that $\beta=1$. In the real case with overlapping, we find that $\beta<1$ and a correction must be applied using Eq.3.

It is also possible to obtain $\beta$ independently using direct calibration measurements involving pure water in only one chamber, independently on ATR profiles. For a combination AB, where A and $\mathrm{B}$ are selected from pure water (W) or an empty volume (E), calibration normalized relative signals are defined as

$$
\rho_{5 / 6}^{\mathrm{AB}}=\frac{S_{5 / 6}^{\mathrm{AB}}-S_{5 / 6}^{\mathrm{EE}}}{S_{5 / 6}^{\mathrm{EE}}} .
$$

Since $\epsilon_{S} \ll 1$, the ratio $r^{\mathrm{SW}} / r^{\mathrm{WW}}$ (see Eq. 2) is developed at the first order in $\epsilon_{S}$. Using Eqs. 3 and 5 , one finds that

$$
\beta=\frac{R_{\mathrm{W}}}{R_{\mathrm{W}}-1}\left[\frac{\rho_{6}^{\mathrm{WE}}}{1+\rho_{6}^{\mathrm{WW}}}-\frac{\rho_{5}^{\mathrm{WE}}}{1+\rho_{5}^{\mathrm{WW}}}\right]
$$


so only two measurements are needed to obtain $\rho_{5 / 6}^{\mathrm{WW}}$ and $\rho_{5 / 6}^{\mathrm{WE}}$, with pure water in one or both chambers. The ATR coefficient $R_{\mathrm{W}}$ for pure water is independently obtained covering the whole prism with distilled water. We measured $R_{\mathrm{W}}=0.54 \pm 0.06$. Using the ATR profiles (Eq. 4), one finally obtains the calibration parameter value $\beta=0.77 \pm 0.07$, while using the direct liquid calibration (Eq. 6) with pure water measurements, we found $\beta=0.68 \pm 0.15$. Both methods provide consistent values of $\beta$, even though the direct liquid calibration is simpler and faster to implement. In the following, an average calibration value of $\beta=0.72 \pm 0.1$ will be used. Once the calibration parameter $\beta$ is known, the measurement of the relative water reflectivity $\epsilon_{S}$ for a given solution is obtained by calculating the ratios $r^{\mathrm{SW}}$ and $r^{\mathrm{WW}}$ from the simultaneous acquisition of the lock-in signals (Eq 2), and then applying the correction from Eq 3.
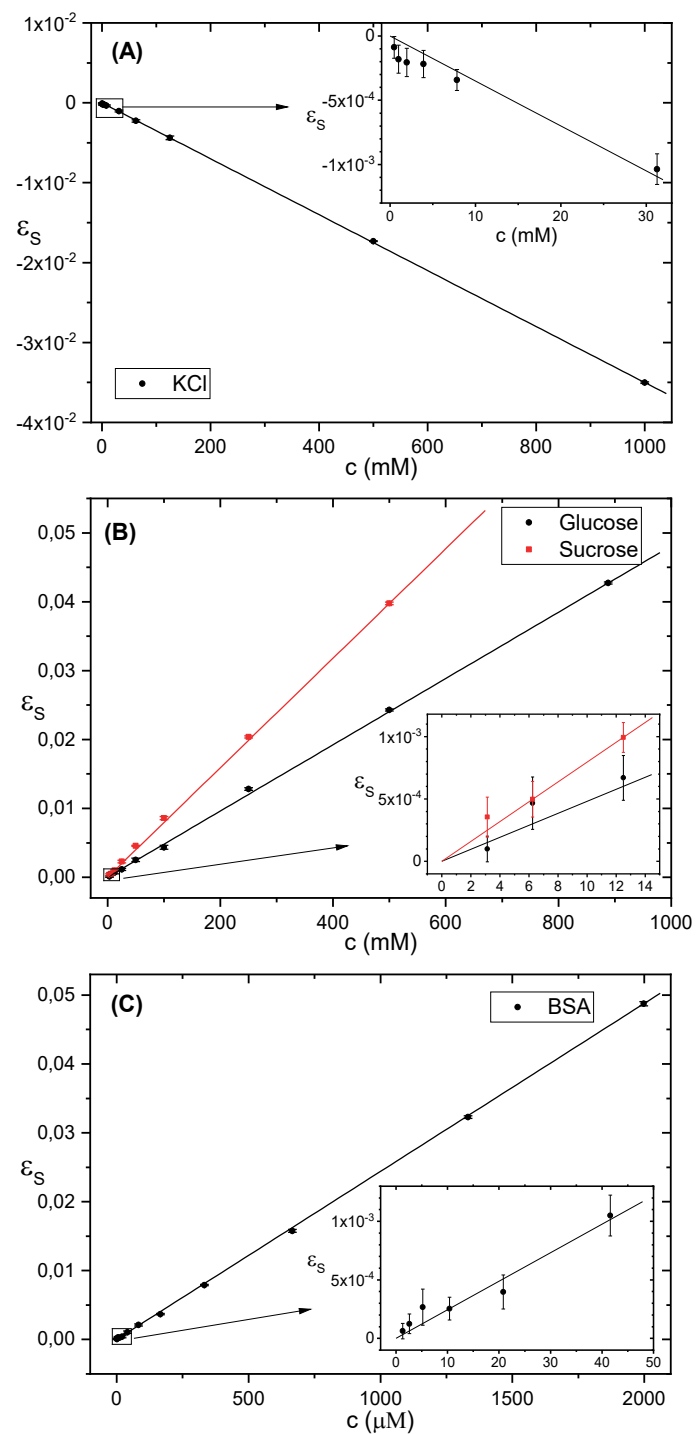

Fig. 3. Relative ATR signals $\epsilon_{S}$ obtained for solutions of $\mathrm{KCl}$ (A), glucose and sucrose (B), and bovine serum albumin (C). Insets show data at low concentrations. Straight lines are linear fits.

The performances of the ATR sensor are demonstrated by measurements on several solutions 
of ions $(\mathrm{KCl}, 74.6 \mathrm{Da})$, sugars (glucose, 180.20 Da, and sucrose, 342.3 Da) and proteins (bovine serum albumine, BSA, $66.5 \mathrm{kDa}$ ), over a wide range of concentrations. For BSA, the molecules were weighed and dissolved in ultrapure water by slow agitation. They were filtered with $0.2 \mu \mathrm{m}$ pore filters, and their final concentration was determined by UV spectrophotometry (NanoDrop 2000c) with the reference molar extinction data. This guarantees well-dissolved solutions at high concentrations. For each solution, the ratio $r^{S W}=S_{6}^{S W} / S_{5}^{S W}$ is calculated as the average of at least 5 independent 200 s-measurements, and compared to the ratio $r^{W W}$ for distilled water. The normalized ATR signals $\epsilon_{S}$ in function of the molar concentration c are shown in Fig 3, after calibration adjustment $(\beta=0.72)$. The inset figures focus on values at low concentrations. For each solute, the concentration dynamic range exceeds 3 orders of magnitude, and the signal is linear with concentration. As expected, we observe that $\epsilon_{S}$ is always negative for $\mathrm{KCl}$ (Fig 3A) and always positive for sugar (Fig 3B) and BSA (Fig 3C) solutions. This originates from the fundamental role of the hydration shell around solutes, whose influence is proportionally more important for small charged molecules such as ions in $\mathrm{KCl}$ [1]. This opposite slope is clearly observed in the relative dielectric constants of these solutes $[8,15,25,26]$. Very low concentrations are detectable, such as $500 \mu \mathrm{M}$ for $\mathrm{KCl}$ or $1 \mu \mathrm{M}$ for BSA, which represents a significant step towards the detection of very low concentrations.

In conclusion, we described a new precise and stable terahertz sensor based on a differential ATR configuration, reaching a relative sensitivity of $10^{-4}$ and a micro-molar level of detection of molecules in solution. The same principle of differential dual modulation could also be applied to broadband spectroscopy, such as time domain spectroscopy or tunable sources by simply changing the source. This sensor is of particular interest for the physicochemical study of molecule solvation and the detection of very low concentrations of molecules, including biomolecules. This device with high long-term stability is also well suited for microfluidic circuits and biological applications.

Funding. Agence Nationale de la Recherche (ANR-11-EQPX-0029).

Disclosures. The authors declare no conflicts of interest.

Data availability. Data underlying the results presented in this paper are not publicly available at this time but may be obtained from the authors upon reasonable request.

\section{References}

1. M. Heyden, D. J. Tobias, and D. V. Matyushov, J Chem Phys 137, 235103 (2012).

2. O. A. Smolyanskaya, N. V. Chernomyrdin, A. A. Konovko et al., Prog. Quantum Electron. 62, 1-77 (2018).

3. L. Zhang, L. Wang, Y.-T. Kao et al., Proc Natl Acad Sci U S A 104, 18461-18466 (2007).

4. A. Azan, M. Grognot, T. García-Sánchez et al., Sci. reports 10, 10471 (2020).

5. X. Zheng and G. Gallot, Biophys. J. 119, 749-755 (2020).

6. K. Shiraga, Y. Ogawa, T. Suzuki et al., J. Infrared Millim. Terahertz Waves 35, 493-502 (2014).

7. A. Charkhesht, C. K. Regmi, K. R. Mitchell-Koch et al., J Phys Chem B 122, 6341-6350 (2018).

8. K. Shiraga, Y. Ogawa, and N. Kondo, Biophys J 111, 2629-2641 (2016).

9. Y. Sun, J. Zhong, C. Zhang et al., J Biomed Opt 20, 037006 (2015).

10. S. Ebbinghaus, S. J. Kim, M. Heyden et al., Proc Natl Acad Sci U S A 104, 20749-52 (2007).

11. B. Ng, J. Wu, S. M. Hanham et al., Adv. Opt. Mater. 1, 543-548 (2013).

12. A. Wojdyla and G. Gallot, Opt. Lett. 38, 112-114 (2013).

13. M. Grognot and G. Gallot, Appl. Phys. Lett. 107, 103702 (2015).

14. M. Tang, M. Zhang, L. Xia et al., Biomed Opt Express 11, 5362-5372 (2020).

15. J. A. Morales-Hernandez, A. K. Singh, S. J. Villanueva-Rodriguez et al., Food Chem 291, 94-100 (2019).

16. J. W. Bye, S. Meliga, D. Ferachou et al., J Phys Chem A 118, 83-8 (2014).

17. K. Serita, E. Matsuda, K. Okada et al., APL Photonics 3 (2018).

18. P. A. George, W. Hui, F. Rana et al., Opt Express 16, 1577-1582 (2008).

19. A. Soltani, H. Neshasteh, A. Mataji-Kojouri et al., Appl. Phys. Lett. 108 (2016).

20. T. Kiwa, T. Kamiya, T. Morimoto et al., Opt Express 26, 8232-8238 (2018).

21. D. Jahn, A. Soltani, J. C. Balzer et al., J. Appl. Phys. 121 (2017).

22. M.Nagel, F. Richter, P. Haring-Bolivar et al., Phys. Med. Biol. 48, 3625-3636 (2003).

23. C. Rønne, L. Thrane, P.-O. Åstrand et al., J. Chem. Phys. 107, 5319-5331 (1997). 
24. M. Born and E. Wolf, Principles of optics 6th Edition (Cambridge University Press, Cambridge, 1997).

25. M. Grognot and G. Gallot, J. Phys. Chem. B 121, 9508-9512 (2017).

26. M. L. T. Asaki, A. Redondo, T. A. Zawodzinski et al., J. Chem. Phys. 116, 8469-8482 (2002). 